\title{
DISPERSION OF MAGNETIC FIELDS IN MOLECULAR CLOUDS. III.
}

\author{
Martin Houde $^{1,2}$, Ramprasad RaO ${ }^{3}$, John E. Vaillancourt ${ }^{4}$, and Roger H. Hildebrand ${ }^{5,6}$ \\ ${ }^{1}$ Department of Physics and Astronomy, The University of Western Ontario, London, ON, N6A 3K7, Canada \\ ${ }^{2}$ Division of Physics, Mathematics and Astronomy, California Institute of Technology, Pasadena, CA 91125, USA \\ ${ }^{3}$ Academia Sinica Institute of Astronomy and Astrophysics, Taipei, Taiwan \\ ${ }^{4}$ Stratospheric Observatory for Infrared Astronomy, Universities Space Research Association, NASA Ames Research Center, Moffet Field, CA 94035, USA \\ ${ }^{5}$ Department of Astronomy and Astrophysics and Enrico Fermi Institute, The University of Chicago, Chicago, IL 60637, USA \\ ${ }^{6}$ Department of Physics, The University of Chicago, Chicago, IL 60637, USA \\ Received 2010 October 05; accepted 2011 March 24; published 2011 May 12
}

\begin{abstract}
We apply our technique on the dispersion of magnetic fields in molecular clouds to high spatial resolution Submillimeter Array polarization data obtained for Orion KL in OMC-1, IRAS 16293, and NGC 1333 IRAS 4A. We show how one can take advantage of such high-resolution data to characterize the magnetized turbulence power spectrum in the inertial and dissipation ranges. For Orion KL we determine that in the inertial range the spectrum can be approximately fitted with a power law $k^{-(2.9 \pm 0.9)}$ and we report a value of $9.9 \mathrm{mpc}$ for $\lambda_{\mathrm{AD}}$, the high spatial frequency cutoff presumably due to turbulent ambipolar diffusion. For the same parameters we have $\sim k^{-(1.4 \pm 0.4)}$ and a tentative value of $\lambda_{\mathrm{AD}} \simeq 2.2 \mathrm{mpc}$ for NGC 1333 IRAS 4A, and $\sim k^{-(1.8 \pm 0.3)}$ with an upper limit of $\lambda_{\mathrm{AD}} \lesssim 1.8 \mathrm{mpc}$ for IRAS 16293 . We also discuss the application of the technique to interferometry measurements and the effects of the inherent spatial filtering process on the interpretation of the results.
\end{abstract}

Key words: ISM: clouds - ISM: magnetic fields - polarization - turbulence

\section{INTRODUCTION}

The fact that the relative importance of magnetic fields and turbulence in the process of star formation is still a matter of debate (Mouschovias \& Tassis 2009; Crutcher et al. 2010) can be traced to the many difficulties of probing magnetic fields in molecular clouds. While the Zeeman effect still provides the only direct way of measuring the strength of (generally the line-of-sight component of) magnetic fields (Heiles 1997; Crutcher et al. 1999; Brogan \& Troland 2001; Falgarone et al. 2008), its weakness in the interstellar medium limits the types of environments and number of regions where detections can successfully be obtained. Without the existence of a prolific technique for measuring the strength of all components of the magnetic field vector, it is difficult to precisely quantify its importance. It is therefore imperative to keep seeking new ideas and techniques that could provide information, even partially, on the nature of magnetic fields. Interestingly, such newly introduced methods of observation or analyses that have been recently put forth for the study of the magnetic field do so by taking advantage of its interplay with turbulence (Houde et al. 2000; Li \& Houde 2008; Heyer et al. 2008).

In this paper, we continue our previous work on the characterization of magnetized turbulence in molecular clouds using polarization maps. In Hildebrand et al. (2009, hereafter Paper I), we showed how the turbulent to ordered magnetic field strength ratio can be evaluated through the structure function of the polarization angles (i.e., the dispersion function) without assuming any model for the ordered component of the magnetic field. Subsequently in Houde et al. (2009, hereafter Paper II), we generalized this analysis by including the process of signal integration through the thickness of the cloud and across the telescope beam. An important development of Paper II was the determination of the magnetized turbulent correlation length scale, which is in effect a measure for the width of the magnetized turbulence power spectrum. We go further in this paper by taking advantage of high-resolution polarization maps to characterize the magnetized turbulence power spectrum in the inertial and dissipation ranges.

We will start in Section 2 with a brief review of the material presented in Paper II on the cloud- and beam-integrated dispersion function that is necessary for our analysis. In Section 3 we present the data on which we will perform our analysis: high-resolution Submillimeter Array (SMA) polarization data of Orion KL (similar to those used for the map presented in Figure 2(c) of Tang et al. 2010), as well as the previously published SMA data of NGC 1333 IRAS 4A (Girart et al. 2006) and IRAS 16293 (Rao et al. 2009). We present the results of our dispersion function analysis in Section 4, where we emphasize some important considerations that are specific to interferometry data. We then follow up with a discussion on the limitations of our technique, improvements that could be implemented, and future applications in Section 5. We end with a short summary in Section 6. Finally, the Appendix pertaining to some data processing aspects can be found at the end of the paper.

\section{THE MAGNETIZED TURBULENCE POWER SPECTRUM}

Following the analysis of Papers I and II, we model the dispersion of the difference $\Delta \Phi(\ell) \equiv \Phi(\mathbf{x})-\Phi(\mathbf{x}+\ell)$ in the polarization angle $\Phi$ measured at two positions separated by a distance $\ell$ with

$$
\langle\cos [\Delta \Phi(\ell)]\rangle=\frac{\langle\overline{\mathbf{B}} \cdot \overline{\mathbf{B}}(\ell)\rangle}{\langle\overline{\mathbf{B}} \cdot \overline{\mathbf{B}}(0)\rangle},
$$

where $\langle\cdots\rangle$ denotes an average and $\ell=|\boldsymbol{\ell}|$ and $\langle\overline{\mathbf{B}} \cdot \overline{\mathbf{B}}(\ell)\rangle \equiv$ $\langle\overline{\mathbf{B}}(\mathbf{r}) \cdot \overline{\mathbf{B}}(\mathbf{r}+\boldsymbol{\ell})\rangle$ (see below). The cloud- and beam-integrated magnetic field is defined with

$$
\overline{\mathbf{B}}(\mathbf{r})=\iint H(\mathbf{r}-\mathbf{a})\left[\frac{1}{\Delta} \int_{0}^{\Delta} F(\mathbf{a}, z) \mathbf{B}(\mathbf{a}, z) d z\right] d^{2} a,
$$


where the beam profile is denoted by $H(\mathbf{r})$, while the weighting function $F(\mathbf{a}, z) \geqslant 0$ is the polarized emission associated with the magnetic field $\mathbf{B}(\mathbf{a}, z)$, and $\Delta$ is the maximum depth of the cloud along any line of sight. The quantity $\mathbf{r}$ is the twodimensional polar radius vector on the plane of the sky and $z$ the depth within the cloud. That is, the position vector in the cloud is given by

$$
\mathbf{x}=r \mathbf{e}_{r}+z \mathbf{e}_{z}
$$

with $\mathbf{e}_{r}$ and $\mathbf{e}_{z}$ the unit basis vectors along $\mathbf{r}$ and the $z$-axis (which is oriented along the line of sight), respectively. The distance $\ell$ in Equation (1) is also confined to the plane of the sky. We assume that the magnetic field $\mathbf{B}(\mathbf{x})$ is composed of an ordered field, $\mathbf{B}_{0}(\mathbf{x})$, and a turbulent (random), zero-mean component, $\mathbf{B}_{\mathrm{t}}(\mathbf{x})$, such that

$$
\mathbf{B}(\mathbf{x})=\mathbf{B}_{0}(\mathbf{x})+\mathbf{B}_{\mathrm{t}}(\mathbf{x}) .
$$

For simplicity, we assume the polarized flux $F(\mathbf{x})$ to be composed of an ordered component only (the more general case where a turbulent component is added was discussed in Paper II). Stationarity, homogeneity, and isotropy (see Section 5.2) in the magnetic field strength were assumed for Equation (1), while statistical independence between ordered and turbulent components will also be implied in what follows.

Following the analysis of Paper II, it is found that the dispersion function $1-\langle\cos [\Delta \Phi(\ell)]\rangle$ can be broken up into turbulent and ordered terms

$$
\begin{aligned}
1-\langle\cos [\Delta \Phi(\ell)]\rangle & =\left[b^{2}(0)-b^{2}(\ell)\right]+\left[\alpha^{2}(0)-\alpha^{2}(\ell)\right] \\
& =\left\{b^{2}(0)+\left[\alpha^{2}(0)-\alpha^{2}(\ell)\right]\right\}-b^{2}(\ell)
\end{aligned}
$$

with the ordered and turbulent autocorrelation functions given by

$$
\begin{aligned}
\alpha^{2}(\ell) & =\frac{\left\langle\overline{\mathbf{B}}_{0} \cdot \overline{\mathbf{B}}_{0}(\ell)\right\rangle}{\langle\overline{\mathbf{B}} \cdot \overline{\mathbf{B}}(0)\rangle}, \\
b^{2}(\ell) & =\frac{\left\langle\overline{\mathbf{B}}_{\mathrm{t}} \cdot \overline{\mathbf{B}}_{\mathrm{t}}(\ell)\right\rangle}{\langle\overline{\mathbf{B}} \cdot \overline{\mathbf{B}}(0)\rangle},
\end{aligned}
$$

respectively. The ordered function $\alpha^{2}(0)-\alpha^{2}(\ell)$ can be advantageously modeled with a Taylor series

$$
\alpha^{2}(0)-\alpha^{2}(\ell)=\sum_{j=1}^{\infty} a_{2 j} \ell^{2 j}
$$

while $b^{2}(0)$ is simply the turbulent to total magnetic energy ratio (that is, for the corresponding cloud- and beam-integrated quantities). As was shown in Papers I and II, the quantity within the curly braces in Equation (5) can be readily determined from polarization maps by calculating the dispersion function (i.e., the left-hand side of that equation) from the data and fitting

$$
b^{2}(0)+\left[\alpha^{2}(0)-\alpha^{2}(\ell)\right]=b^{2}(0)+\sum_{j=1}^{\infty} a_{2 j} \ell^{2 j}
$$

to the data outside of the region where $b^{2}(\ell)$ is dominant (i.e., at lower values of $\ell$ ). Once Equation (9) is evaluated the (normalized) turbulent cloud- and beam-integrated autocorrelation function $b^{2}(\ell)$ can be extracted from the data through Equation (5).
Alternatively, the integrated turbulent autocorrelation function $b^{2}(\ell)$ can also be analytically derived using (see Equation (A5) of Paper II)

$$
\begin{aligned}
\left\langle\overline{\mathbf{B}}_{\mathrm{t}} \cdot \overline{\mathbf{B}}_{\mathrm{t}}(\boldsymbol{\ell})\right\rangle= & \iiint \int H(\mathbf{a}) H\left(\mathbf{a}^{\prime}+\boldsymbol{\ell}\right) \\
& \times\left[\frac{2}{\Delta} \int_{0}^{\Delta}\left(1-\frac{u}{\Delta}\right) \mathcal{R}_{3 \mathrm{D}, \mathrm{t}}(v, u) d u\right] d^{2} a^{\prime} d^{2} a
\end{aligned}
$$

with $\mathcal{R}_{3 \mathrm{D}, \mathrm{t}}(v, u)=\left\langle F(\mathbf{a}, z) F\left(\mathbf{a}^{\prime}, z^{\prime}\right)\right\rangle\left\langle\mathbf{B}_{\mathrm{t}}(\mathbf{a}, z) \cdot \mathbf{B}_{\mathrm{t}}\left(\mathbf{a}^{\prime}, z^{\prime}\right)\right\rangle, u=$ $\left|z^{\prime}-z\right|$ and $v=\left|\mathbf{a}^{\prime}-\mathbf{a}\right|$, and a similar equation for $\langle\overline{\mathbf{B}} \cdot \overline{\mathbf{B}}(0)\rangle$. Since we are mostly interested in determining the shape of the magnetized turbulence power spectrum, we will concentrate on the Fourier transform of $b^{2}(\ell)$ (see Equation (A12) of Paper II)

$b^{2}\left(\mathbf{k}_{v}\right)=\frac{1}{\left\langle\bar{B}^{2}\right\rangle}\left\|H\left(\mathbf{k}_{v}\right)\right\|^{2}\left[\int \mathcal{R}_{3 \mathrm{D}, \mathrm{t}}\left(\mathbf{k}_{v}, k_{u}\right) \operatorname{sinc}^{2}\left(\frac{k_{u} \Delta}{2}\right) d k_{u}\right]$,

where $\left\langle\bar{B}^{2}\right\rangle \equiv\langle\overline{\mathbf{B}} \cdot \overline{\mathbf{B}}(0)\rangle$ and the Fourier transform of a function is represented by simply replacing the spatial arguments by their k-space counterparts (e.g., $\left.\mathcal{R}_{3 \mathrm{D}, \mathrm{t}}(v, u) \rightleftharpoons \mathcal{R}_{3 \mathrm{D}, \mathrm{t}}\left(\mathbf{k}_{v}, k_{u}\right)\right)$. Equation (11) can be redefined with

$$
b^{2}\left(\mathbf{k}_{v}\right)=\left\|H\left(\mathbf{k}_{v}\right)\right\|^{2} \frac{\mathcal{R}_{\mathrm{t}}\left(\mathbf{k}_{v}\right)}{\left\langle\bar{B}^{2}\right\rangle},
$$

where $\mathcal{R}_{\mathrm{t}}\left(\mathbf{k}_{v}\right) \equiv \int \mathcal{R}_{3 \mathrm{D}, \mathrm{t}}\left(\mathbf{k}_{v}, k_{u}\right) \operatorname{sinc}^{2}\left(k_{u} \Delta / 2\right) d k_{u}$ is now interpreted as the two-dimensional turbulence power spectrum we seek to evaluate. We will accomplish this by taking the Fourier transform of $b^{2}(\ell)$ obtained from the data, as explained in the discussion following Equation (9) above, to evaluate $b^{2}\left(\mathbf{k}_{v}\right)$ on the left-hand side of Equation (12) and then invert this relation to determine $\mathcal{R}_{\mathrm{t}}\left(\mathbf{k}_{v}\right) /\left\langle\bar{B}^{2}\right\rangle$ (with a Wiener filter to remove the filtering due to $\left\|H\left(\mathbf{k}_{v}\right)\right\|^{2}$; see the Appendix).

\section{OBSERVATIONS}

The observations for Orion KL were carried out on 2006 September 10 and 2008 January 6 using the SMA (Ho et al. $2004)^{7}$ in the compact array configuration, with the projected baseline lengths ranging from 15 to $80 \mathrm{k} \lambda(\lambda=870 \mu \mathrm{m})$. The phase center is at R.A.(J2000) $=5^{\mathrm{h}} 35^{\mathrm{m}} 14.5$, decl.(J2000) $=$ $-5^{\circ} 22^{\prime} 30^{\prime} .4$. The SMA receiver system has two sidebands, each with a bandwidth of $\sim 2 \mathrm{GHz}$. The sampled sky frequencies range from 345.5 to $347.5 \mathrm{GHz}$ in the upper sideband and from 335.5 to $337.5 \mathrm{GHz}$ in the lower, with a uniform spectral resolution of $0.812 \mathrm{MHz}$ (corresponding to a velocity resolution of $0.7 \mathrm{~km} \mathrm{~s}^{-1}$ ). At these frequencies, the primary beam size (or field of view) of the SMA is $\sim 32^{\prime \prime}$. Within the observational bandwidth, there is a significant contribution to the total emission from spectral lines of a number of molecular transitions (notably $\mathrm{CO}(J=3 \rightarrow 2)$ and $\mathrm{SiO}(J=8 \rightarrow 7)$ ), and the continuum is generated after removing the spectral line contamination. Using natural weighting of the visibilities, the synthesized beam size is $2^{\prime \prime} .6 \times 1^{\prime \prime}$. 7 . The noise level in the Stokes $I$ image is $\sim 0.3 \mathrm{Jy}$ beam $^{-1}$. This is much higher than the theoretical noise level due to the limited dynamic range in the Stokes $I$ map.

\footnotetext{
7 The Submillimeter Array is a joint project between the Smithsonian Astrophysical Observatory and the Academia Sinica Institute of Astronomy and Astrophysics and is funded by the Smithsonian Institution and the Academia Sinica.
} 
The noise levels of both the Stokes $Q$ and $U$ images, which are much fainter, are much closer to the theoretical noise level, at $10 \mathrm{mJy}$ beam $^{-1}$. Our observations for Orion KL have much data in common with those used for the polarization map presented in Figure 2(c) of Tang et al. (2010), which we refer the reader to in view of its similarity to the map that can be derived from our data. We note, however, that our data have a slightly higher spatial resolution than the 2 ." $8 \times 1$ 1". 8 synthesized beam size of Tang et al. (2010). Koch et al. (2010) also performed a dispersion analysis on the Tang et al. (2010) map based on our Papers I and II. Importantly, for our analysis, we use all polarization vectors available at the sampling rate of 0 '. 25 , provided they satisfy the condition $p \geqslant 3 \sigma_{p}$, with $p$ and $\sigma_{p}$ the polarization level and its uncertainty, respectively. This implies that many of our data points are correlated with each other since several are contained with a single synthesized beam profile. This is beneficial for our analysis as it allows for a better fit of Equation (9) from the dispersion data. The correlation between data points is also accounted for in the evaluation of the dispersion function and its uncertainty as a function of $\ell$ (see Appendix B of Paper II).

The data were calibrated and processed using the software package MIRIAD (Wright \& Sault 1993). The gain calibration was obtained from observations of the QSO $0528+134$. It is necessary to remove the contributions due to instrumental polarization as these are roughly similar in magnitude to the observed source polarization and can corrupt the data (see Marrone et al. 2006 and Marrone \& Rao 2008 for the details of this method). The instrumental polarization was obtained from observations of the strong quasar 3c273 for $2 \mathrm{hr}$ during transit. The total intensity (Stokes $I$ ) map was deconvolved using the task CLEAN in MIRIAD. We derived the polarized intensity $\left(I_{\mathrm{p}}\right)$ and position angles (P.A.s) with the task IMPOL, also using the CLEANed Stokes $Q$ and $U$ maps. The task IMPOL further removed the effects of the bias of the positive measure of $I_{\mathrm{p}}$.

The data for IRAS 16293 and NGC 1333 IRAS 4A were also obtained at the SMA and reduced in a similar manner. They were previously published and described in detail in Rao et al. (2009) and Girart et al. (2006), respectively. Their synthesized beam sizes and sampling rates are $3^{\prime \prime} .1 \times 22^{\prime \prime} .0$ and 0 .' 25 for IRAS 16293, and 1". $6 \times 1^{\prime \prime} .0$ and 0.2 for NGC 1333 IRAS 4A.

\section{RESULTS}

\subsection{Dispersion Functions from Interferometry Data-Orion $K L$}

Although the discussion that follows applies equally well to all the data presented in this paper, we will refer only to the Orion KL data (see the map of Figure 2(c) of Tang et al. 2010) in this sub-section in order to discuss the application of the dispersion analysis to interferometry data. One of our main goals here is to emphasize that there are important differences with results obtained with interferometry in relation to what one would get with single-dish data.

We first note that because of the unavoidable $180^{\circ}$ ambiguity when determining the orientation of the magnetic field from polarization data, one has to be careful when proceeding with a dispersion analysis of polarization angles. Indeed, any such analysis can only be successfully carried out in regions of polarization maps for which changes in polarization angles with position are sufficiently smooth to ensure, at least to a reasonable degree, that there are no possible reversals in the field direction. For this reason, we have excluded from our analysis a small isolated "clump" located at an offset of $\Delta \alpha \approx 8^{\prime \prime}$ and
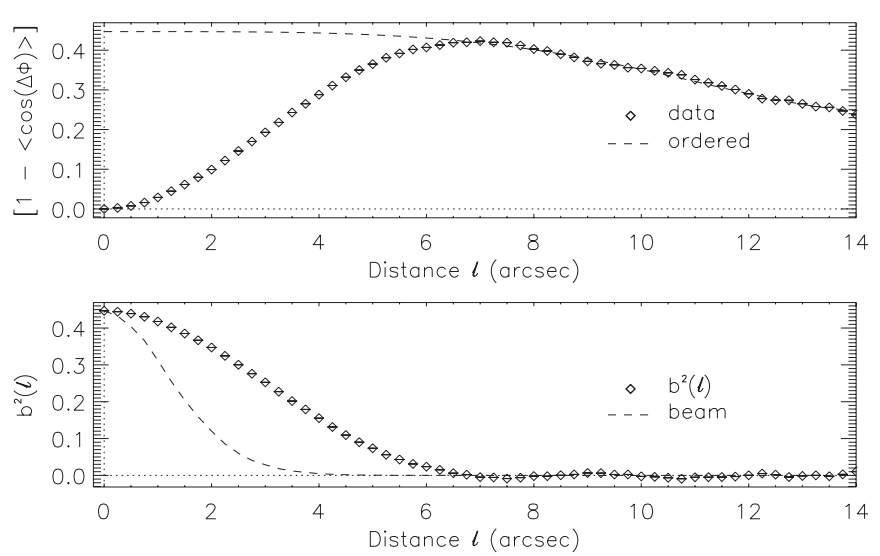

Figure 1. Top: the dispersion function $1-\langle\cos [\Delta \Phi(\ell)]\rangle$ for Orion KL. The broken curve ("ordered") is the fit for the sum of the turbulent to total magnetic energy ratio $b^{2}(0)$ and the ordered component $\sum_{j=1}^{3} a_{2 j} \ell^{2 j}$ implicit to the data (symbols) using the points, where $\ell \geqslant 7^{\prime \prime}$. Both functions are plotted as a function of $\ell$. Bottom: the turbulent autocorrelation function $b^{2}(\ell)$ (symbols), as obtained by subtracting the data points to the "ordered" curve in the top graph, while the broken curve shows the radial profile of the "mean autocorrelated synthesized beam" (see the text).

$\Delta \delta \approx 11^{\prime \prime}$ to the north of position of peak intensity of Orion KL (i.e., at $\alpha(\mathrm{J} 2000) \simeq 5^{\mathrm{h}} 35^{\mathrm{m}} 14.9$ and $\delta(\mathrm{J} 2000) \simeq-5^{\circ} 22^{\prime} 17^{\prime \prime}$ in Figure 2(c) of Tang et al. 2010).

Following the analysis done in Paper II, the dispersion function $1-\langle\cos [\Delta \Phi(\ell)]\rangle$ and turbulent autocorrelation function $b^{2}(\ell)$ determined from the Orion KL polarization map are shown as a function of the distance $\ell$ in Figure 1 (symbols in top and bottom graphs, respectively). It is important to note that although these functions are only plotted for $\ell \geqslant 0$, the data are actually two-dimensional in nature and exhibit cylindrical symmetry (in the plane containing $\ell$ about the $\ell=0$ axis) because of the assumed isotropy of the dispersion function. With the prescription given in Paper I (or Paper II) one then fits the (sum of the) turbulent to total magnetic energy ratio $b^{2}(0)$ and the ordered magnetic field component $\sum_{j} a_{2 j} \ell^{2 j}$, for which we use the lowest order polynomial that fits the data, with the broken curve shown in the top graph (see Equation (9)); the subtraction of the data from that curve would then yield $b^{2}(\ell)$ from which the analysis could proceed (see Equation (5)). Although this is perfectly adequate for single-dish data, an important fact needs to be emphasized when dealing with interferometry data.

The aforementioned subtraction of the dispersion data from the broken curve in the top graph of Figure 1 leads to turbulent autocorrelation data that satisfy $b^{2}(\ell) \geqslant 0$ for most if not all values of $\ell$, which also implies, of course, that the integral of that function over space will be positive. But this should not be possible with a polarization map obtained with an interferometer, as can be assessed from Equation (12), since

$$
\begin{aligned}
2 \pi \int_{0}^{\infty} b^{2}(\ell) \ell d \ell & =b^{2}\left(\mathbf{k}_{v}=0\right) \\
& =\frac{1}{\left\langle\bar{B}^{2}\right\rangle}\left[\left\|H\left(\mathbf{k}_{v}\right)\right\|^{2} \mathcal{R}_{\mathrm{t}}\left(\mathbf{k}_{v}\right)\right]_{\mathbf{k}_{v}=0} \\
& =0
\end{aligned}
$$

because $H\left(\mathbf{k}_{v}=0\right)=0$ for the so-called dirty beam of an interferometer. This, of course, is directly related to the wellknown missing-flux "issue" that is implicit to interferometry 
data. But importantly, it is also contrary to single-dish data where $H\left(\mathbf{k}_{v}=0\right)=1$, when inefficiencies are accounted for. It therefore follows that an acceptable fit $b^{2}(0)+\sum_{j} a_{2 j} \ell^{2 j}$ to our dispersion function interferometry data should satisfy the condition

$$
2 \pi \int_{0}^{\infty} b^{2}(\ell) \ell d \ell=0 .
$$

Although we have restricted the condition of Equation (15) to the turbulent component $b^{2}(\ell)$, it also applies equally well to the ordered component and the total normalized autocorrelation function of the magnetic field given by Equation (1). The important point we need to acknowledge is that it is impossible to know exactly what correct fit for the turbulent to total magnetic energy ratio and ordered component (i.e., $b^{2}(0)+\sum_{j} a_{2 j} \ell^{2 j}$ ) applies to our Orion KL data (or any other data set) that will verify Equation (15), as there is an infinite number of combinations for $b^{2}(0)$ and $\sum_{j} a_{2 j} \ell^{2 j}$ that will satisfy this condition and no way to discriminate between them. We must therefore accept this as a fundamental limitation to the analysis when dealing with interferometry data alone. This problem can only be avoided if the polarization map includes data that fill the low-frequency portion of the spectrum (including $\mathbf{k}_{v}=0$ ), i.e., when single-dish data are available, since the condition given in Equation (15) will then not apply anymore and the degeneracy on the aforementioned fit will be lifted.

On the other hand, it is perhaps reasonable to expect that our fit for $b^{2}(0)+\sum_{j} a_{2 j} \ell^{2 j}$ (as shown in Figure 1, for example) will affect the shape of the turbulence power spectrum $\mathcal{R}_{\mathrm{t}}\left(\mathbf{k}_{v}\right) /\left\langle\bar{B}^{2}\right\rangle$, which we seek to determine from the dispersion function, mainly at low spatial frequencies. More precisely, the spectral content associated with the fit $b^{2}(0)+\sum_{j} a_{2 j} \ell^{2 j}$ is to a large extent concentrated at low frequencies and will have a diminishing effect on our determination of the shape of the magnetized turbulence power spectrum when moving to higher frequencies. For the purpose of the present analysis, we will proceed by neglecting the condition stated in Equation (15) and perform our study as we normally would for single-dish data, and then discard the low-frequency portion of the turbulent spectrum from our analysis (see below). That is, we adopt the fit shown in the top graph of Figure 1 (i.e., the broken curve) for Orion KL for our analysis. Accordingly, the associated turbulent autocorrelation function $b^{2}(\ell)$ is that shown in the bottom graph of Figure 1 (symbols). Also plotted is the radial profile of the "mean autocorrelated beam" (broken curve). This profile is obtained by first computing the autocorrelation of the synthesized beam, since it is this beam that is used for the polarization map and appears in the expression for the dispersion function (see Equations (1) and (10)), and then averaged azimuthally in the same manner as are the dispersion data. This represents the contribution of the synthesized beam to the (width of) the turbulent autocorrelation function $b^{2}(\ell)$. That is, this is what $b^{2}(\ell)$ would look like in the limit where the intrinsic turbulent correlation length were zero. A comparison of this autocorrelated beam profile to that of $b^{2}(\ell)$ clearly shows the significant contribution of the magnetized turbulence $\mathcal{R}_{\mathrm{t}}(\ell)$ to the overall width and shape of $b^{2}(\ell)$.

The top graph of Figure 2 shows the spectra associated with $b^{2}(\ell)$ (i.e., $b^{2}(k)$ with $k=\left|\mathbf{k}_{v}\right|$; symbols) and the mean autocorrelated synthesized beam (i.e., $\|H(k)\|^{2}$; broken curve) calculated by taking the Fourier transform of the corresponding functions shown in the bottom graph of Figure 1 (see the Appendix). Also shown is the spectrum of the visibility data (or
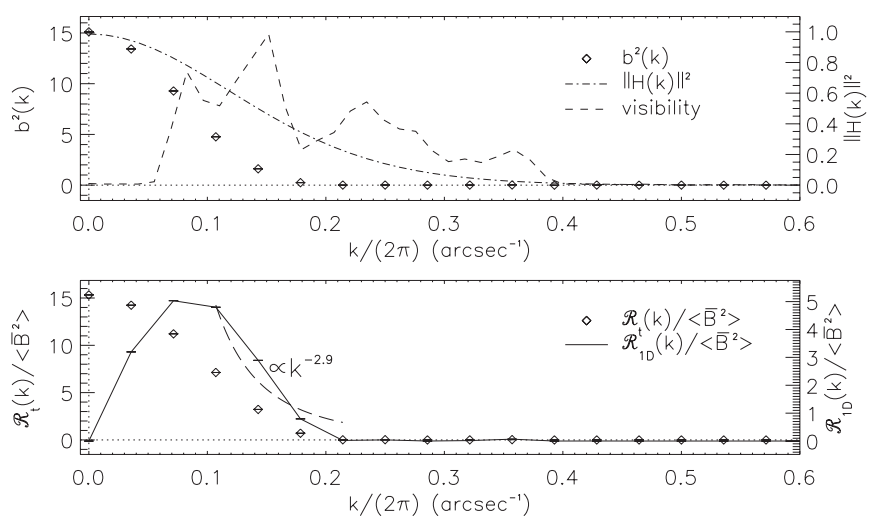

Figure 2. Top: spectra associated to $b^{2}(\ell)$ (i.e., $b^{2}(k)$ with $k=\left|\mathbf{k}_{v}\right|$; symbols) and the mean autocorrelated synthesized beam (i.e., $\|H(k)\|^{2}$ ) calculated by taking the Fourier transform of the corresponding functions shown in the bottom graph of Figure 1. The visibility data (or the spectral profile of the dirty beam) are also shown (see the text) and are normalized to its peak level (using the scale on the right). Bottom: our results for the magnetized turbulence power spectrum profile $\mathcal{R}_{\mathrm{t}}(k) /\left\langle\bar{B}^{2}\right\rangle$ (symbols) and the associated one-dimensional "Kolmogorov-like" spectrum $\mathcal{R}_{1 \mathrm{D}}(k) /\left\langle\bar{B}^{2}\right\rangle$. The broken curve shows an approximate power-law fit $k^{-(2.9 \pm 0.9)}$ to the inertial range.

dirty beam; dot-broken curve), which is normalized to its peak level (using the scale on the right). We can now readily verify that the telescope dirty beam is responsible for the condition stated through Equation (15) since it vanishes at $k=0$, as expected. The fact that our data for $b^{2}(k)$ are non-zero at and close to $k=0$ is a reflection of the fact that, as we discussed above, we cannot precisely evaluate it from the dispersion function. Because of this we exclude the first two points of the spectrum at $k / 2 \pi=0$ and $0.5 \operatorname{arcsec}^{-1}$ from our analysis and concentrate on the rest of the spectrum (i.e., higher frequencies).

The bottom graph of Figure 2 presents the results of our analysis for Orion KL. First, Equation (12) is inverted using a simple Wiener optimal filter (see the Appendix), which is possible since the beam profile $\|H(k)\|^{2}$ is well characterized, to yield the magnetized turbulence power spectrum profile $\mathcal{R}_{\mathrm{t}}(k) /\left\langle\bar{B}^{2}\right\rangle$ (again with $k=\left|\mathbf{k}_{v}\right|$; symbols).

The turbulence power spectrum is not usually expressed with $\mathcal{R}_{\mathrm{t}}(k)$, however, but rather with (Frisch 1995)

$$
\mathcal{R}_{\mathrm{K}}\left(k^{\prime}\right) \equiv 4 \pi k^{\prime 2} \mathcal{R}_{3 \mathrm{D}, \mathrm{t}}\left(k^{\prime}\right),
$$

which is a "one-dimensional" representation of (the threedimensional) $\mathcal{R}_{3 \mathrm{D}, \mathrm{t}}\left(\mathbf{k}_{v}, k_{u}\right)$ with $k^{\prime 2}=\left|\mathbf{k}_{v}\right|^{2}+k_{u}^{2}$. This "Kolmogorov-like" spectrum is particularly well suited for cases of isotropic turbulence. It is unfortunately not possible with our data to recover $\mathcal{R}_{\mathrm{K}}\left(k^{\prime}\right)$ from $\mathcal{R}_{\mathrm{t}}(k)$ because of the integration over $k_{u}$ in Equation (11) that is inherent to the measurement process. On the other hand, it is still possible to define another "one-dimensional" power spectrum $\mathcal{R}_{1 \mathrm{D}}(k)$ with

$$
\mathcal{R}_{1 \mathrm{D}}(k) \equiv 2 \pi k \mathcal{R}_{\mathrm{t}}(k)
$$

which is obtained from our data in a straightforward manner. We show the corresponding result for Orion KL with the solid curve in the bottom graph of Figure 2. Since it is also customary to parameterize the turbulence power spectrum in the inertial range with a power law, we also plot such a fit to $\mathcal{R}_{1 \mathrm{D}}(k) /\left\langle\bar{B}^{2}\right\rangle$ and show that the inertial range approximately scales with $k^{-(2.9 \pm 0.9)}$. Although this result is consistent with theoretical expectations, the small number of spectral points available for the fit and our 


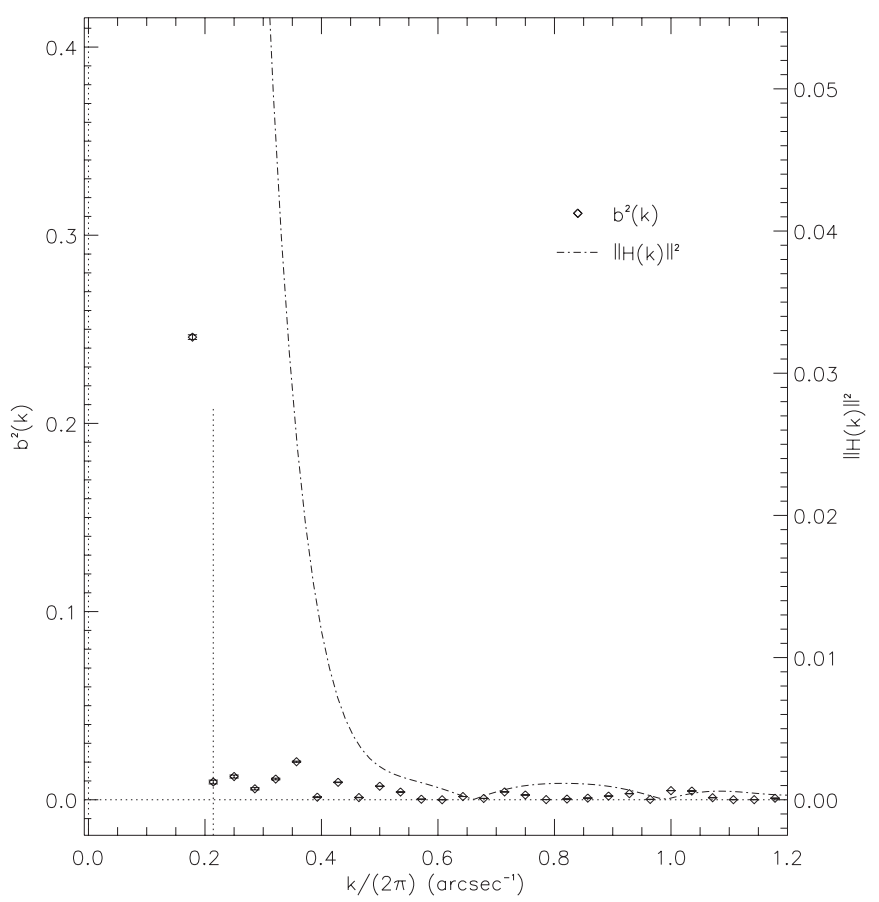

Figure 3. Magnification of the vertical scale of the top graph of Figure 2. For Orion $\mathrm{KL}$ we detect $k_{\mathrm{AD}} / 2 \pi \simeq 0.22 \operatorname{arcsec}^{-1}$ (or $\lambda_{\mathrm{AD}} \simeq 9.9 \mathrm{mpc}$ with an assumed distance of $450 \mathrm{pc}$ ) for the high-frequency spectral cutoff, which is probably due to turbulent ambipolar diffusion (vertical dotted line).

lack of knowledge concerning the precise shape of the ordered component to be subtracted from the dispersion function reduce the robustness of our determination for the spectral index (as is exemplified by the significant uncertainty on its value).

Another important parameter that characterizes the magnetized turbulence power spectrum is the cutoff frequency $k_{\mathrm{AD}}$, or length scale $\lambda_{\mathrm{AD}}=2 \pi / k_{\mathrm{AD}}$, at high frequencies, which is likely due to ambipolar diffusion (Li \& Houde 2008; Hezareh et al. 2010; Lazarian et al. 2004; Falceta-Gonçalves et al. 2010; Tilley $\&$ Balsara 2010; Houde et al. 2011). We find from our results for $\mathcal{R}_{1 \mathrm{D}}(k) /\left\langle\bar{B}^{2}\right\rangle$ in Figure 2 that $k_{\mathrm{AD}} / 2 \pi \sim 0.2 \mathrm{arcsec}^{-1}$. Although this value $\left(\lambda_{\mathrm{AD}} \sim 11 \mathrm{mpc}\right.$, see below) is also consistent with theoretical expectations (Lazarian et al. 2004; Falceta-Gonçalves et al. 2010; Tilley \& Balsara 2010) as well as observationally determined values (Li \& Houde 2008; Hezareh et al. 2010), we should ensure that this spectral cutoff is real and not artificially imposed by data processing or the finite spatial resolution of the interferometer. This can be verified with Figure 3 where we have magnified the vertical scale of the top graph of Figure 2. It is observed that this spectral cutoff is present in the data for $b^{2}(k)$ (i.e., before applying the Wiener filter) and seen to happen well within the bandwidth subtended by the synthesized beam (or rather that of $\left.\|H(k)\|^{2}\right)$, which cuts off at $k / 2 \pi \simeq 0.6 \operatorname{arcsec}^{-1}$. This plot also shows that $k_{\mathrm{AD}} / 2 \pi \simeq 0.22 \mathrm{arcsec}^{-1}$, or alternatively $\lambda_{\mathrm{AD}} \simeq 9.9 \mathrm{mpc}$ for Orion $\mathrm{KL}$ (assumed to be at a distance of $450 \mathrm{pc})$.

\subsection{IRAS 16293}

We applied our dispersion analysis to the polarization map of IRAS 16293 of Rao et al. (2009). The results for the dispersion and turbulent autocorrelation functions, the turbulent spectrum, and the determination of $\lambda_{\mathrm{AD}}$ are shown in Figures 4, 5, and 6 , respectively. The dispersion and turbulent autocorrelation functions (Figure 4) display similar characteristics as those for
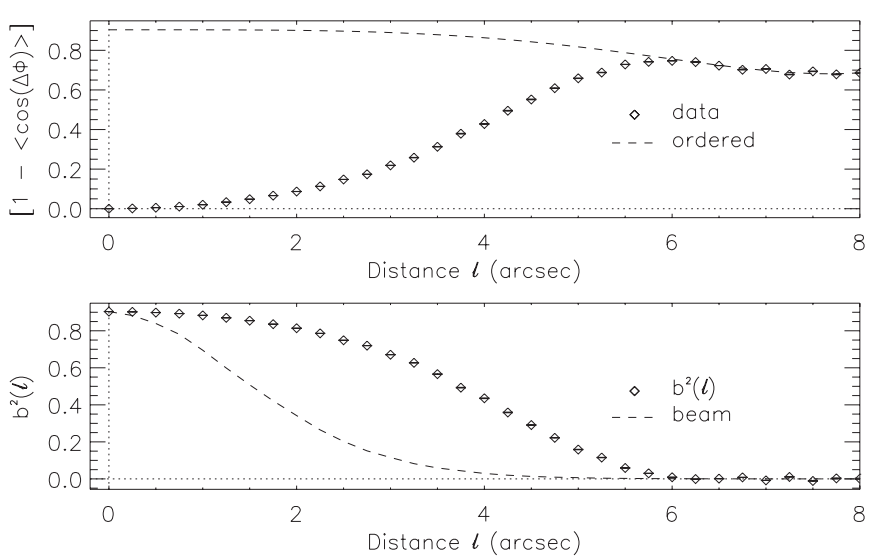

Figure 4. Same as Figure 1 but for IRAS 16293; data points where $\ell \geqslant 6.25$ were used for the fit on the top graph (broken curve).
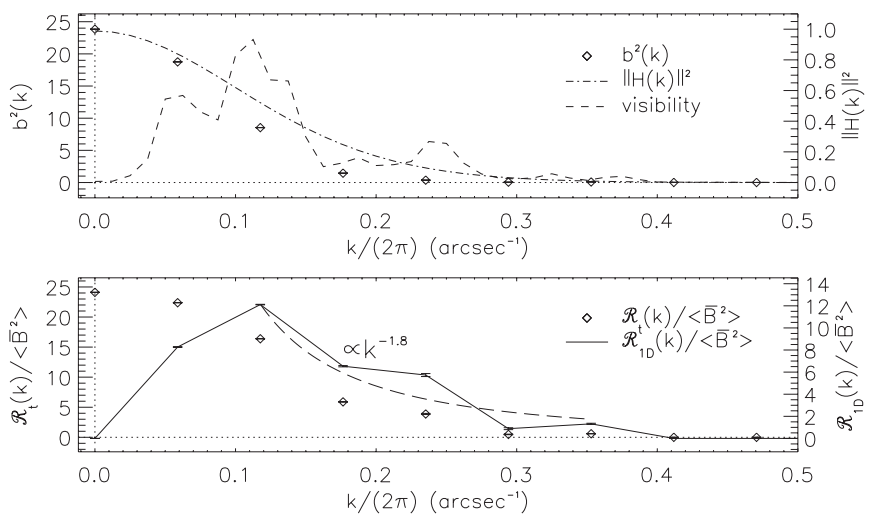

Figure 5. Same as Figure 2 but for IRAS 16293. However, for this source the broken curve on the bottom graph shows an approximate power-law fit $k^{-(1.8 \pm 0.3)}$ to the inertial range.

Orion KL, but the differences are made more obvious when considering the turbulence power spectrum. Indeed, Figure 5 shows that the Kolmogorov-like spectrum for IRAS 16293 scales as $\sim k^{-(1.8 \pm 0.3)}$, while an inspection of Figure 6 makes it clear that the apparent cutoff in the spectrum at $k \simeq 0.4 \operatorname{arcsec}^{-1}$ (vertical dotted line) is likely due to beam filtering. We therefore only report an upper limit of $\lambda_{\mathrm{AD}} \lesssim 1.8 \mathrm{mpc}$ for IRAS 16293 (assumed to be at a distance of $150 \mathrm{pc}$ ) for the high-frequency spectral cutoff due to ambipolar diffusion.

\subsection{NGC 1333 IRAS 4A}

The results of our dispersion analysis as applied to the NGC 1333 IRAS 4A polarization map of Girart et al. (2006) are shown in Figures 7, 8, and 9. For this source the Kolmogorovlike power spectrum is observed to scale with $\sim k^{-(1.4 \pm 0.4)}$ (see Figure 8), while we find $k_{\mathrm{AD}} / 2 \pi \simeq 0.66 \mathrm{arcsec}^{-1}$ (or $\lambda_{\mathrm{AD}} \simeq 2.2 \mathrm{mpc}$ with an assumed distance of $300 \mathrm{pc}$ ) for the high-frequency spectral cutoff due to turbulent ambipolar diffusion (vertical dotted line in Figure 9). However, because of the weakness of $b^{2}(k)$ about $k_{\mathrm{AD}}$, the latter's proximity to the spectral cutoff due to beam filtering, and the aforementioned uncertainty in fitting the ordered component of the turbulent autocorrelation function, we must acknowledge that this estimate for $\lambda_{\mathrm{AD}}$ is tentative. As was the case for Orion KL (and IRAS 16293 for the spectral index), these parameters are consistent with expectations. 


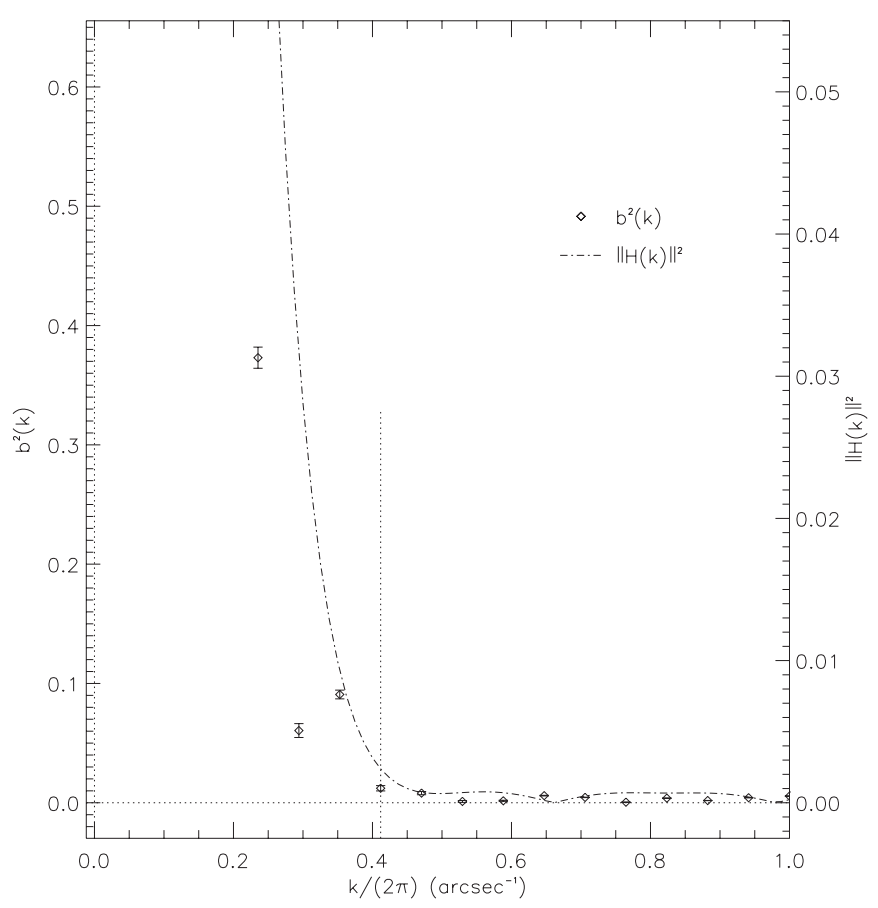

Figure 6. Same as Figure 3 but for IRAS 16293. However, for this source we observe that the high-frequency cutoff present in the data for $b^{2}(k)$ (i.e., before applying the Wiener filter) is correlated with the bandwidth subtended by the synthesized beam $\|H(k)\|^{2}$, which cuts off at $k / 2 \pi \simeq 0.41 \operatorname{arcsec}^{-1}$. We therefore have an upper limit of $\lambda_{\mathrm{AD}} \lesssim 1.8 \mathrm{mpc}$ for IRAS 16293 (assumed distance of $150 \mathrm{pc}$ ) for the high-frequency spectral cutoff expected from turbulent ambipolar diffusion (vertical dotted line).
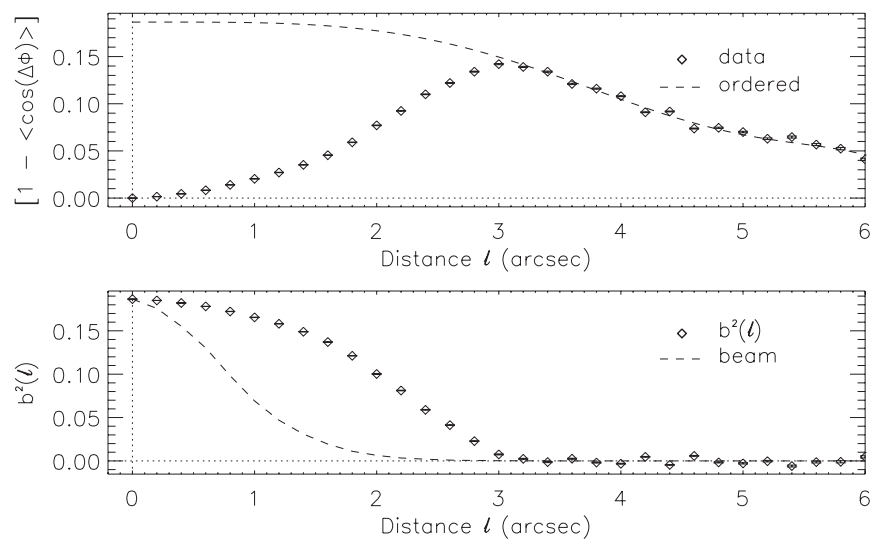

Figure 7. Same as Figure 1 but for NGC 1333 IRAS 4A. The broken curve on the top graph ("ordered") is the fit for the turbulent to total magnetic energy ratio $b^{2}(0)$ and the ordered component $\sum_{j=1}^{4} a_{2 j} \ell^{2 j}$ implicit to the data (symbols) using the points where $\ell \geqslant 3$." 4 .

\section{DISCUSSION}

\subsection{Limitations of the Dispersion Technique}

The high spatial resolution with which the polarization data analyzed in this paper were obtained has allowed us to determine fundamental parameters that characterize the magnetized turbulence power spectrum in some well-known star-forming regions. However, the same observing mode that allows these realizations also brings with it some limitations due to the filtering of low spatial frequencies inherent to interferometry. We already discussed in detail in Section 4 how this impedes the precise determination of the power spectrum at
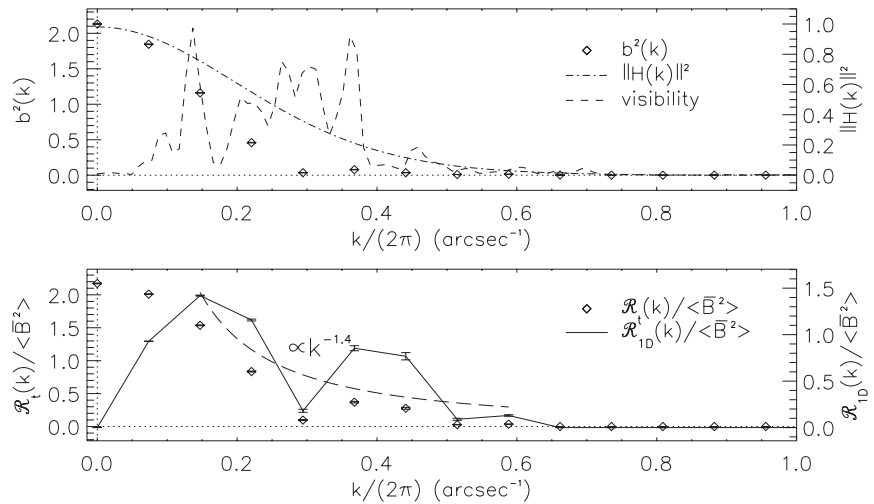

Figure 8. Same as Figure 2 but for NGC 1333 IRAS 4A. However, for this source the broken curve on the bottom graph shows an approximate power-law fit $k^{-(1.4 \pm 0.4)}$ to the inertial range.

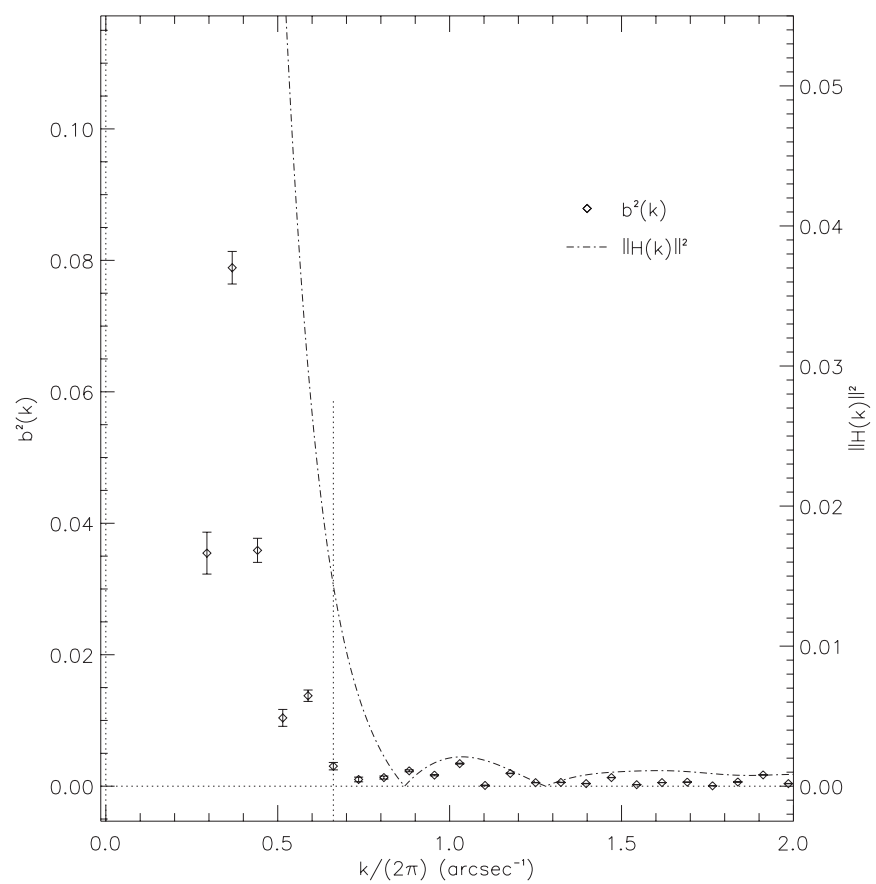

Figure 9. Same as Figure 3 but for NGC 1333 IRAS 4A. However, for this source we tentatively detect $k_{\mathrm{AD}} / 2 \pi \simeq 0.66 \operatorname{arcsec}^{-1}$ (or $\lambda_{\mathrm{AD}} \simeq 2.2 \mathrm{mpc}$ with an assumed distance of $300 \mathrm{pc}$ ) for the high-frequency spectral cutoff, which is probably due to turbulent ambipolar diffusion (vertical dotted line).

low frequencies. We now discuss two more consequences that result from this limitation.

\subsubsection{The Chandrasekhar-Fermi Technique}

As was shown in Paper I, the value for the turbulent to ordered magnetic energy ratio $b^{2}(\ell=0)$ obtained when fitting the dispersion function (see the broken curves in the top graphs of Figures 1, 4, and 7) corresponds to the value that one would normally use for the determination of the ordered magnetic field strength with the Chandrasekhar-Fermi equation (Chandrasekhar \& Fermi 1953). More precisely, we have (see Equations (7) and (8) of Paper I)

$$
\left\langle B_{0}^{2}\right\rangle \simeq 4 \pi \rho\left(\frac{\sigma^{2}(v)}{b^{2}(0)}\right),
$$

where $\rho$ and $\sigma(v)$ are the mass density and the one-dimensional turbulent velocity dispersion, respectively. However, as was 
noted earlier the value of $b^{2}(\ell=0)$ determined from interferometry data alone cannot be precisely determined because of the filtering of low spatial frequencies. An error in the estimate of the ordered magnetic field strength will then follow due to the presence of $b^{2}(\ell=0)$ in the denominator of Equation (18). Although such an error will also arise with single-dish data (where the filtering happens instead at high frequencies), the relative importance of this error in comparison to what is expected with interferometry observations can be studied by considering the Fourier transform that links $b^{2}(\ell)$ to $b^{2}\left(\mathbf{k}_{v}\right)$

$$
b^{2}(\ell)=\frac{1}{(2 \pi)^{2}} \iint b^{2}\left(\mathbf{k}_{v}\right) e^{\ell \cdot \mathbf{k}_{v}} d^{2} k_{v} .
$$

It follows from this and Equation (12) that

$$
\begin{aligned}
b^{2}(\ell=0) & =\frac{1}{(2 \pi)^{2}} \iint b^{2}\left(\mathbf{k}_{v}\right) d^{2} k_{v} \\
& =\frac{1}{(2 \pi)^{2}\left\langle\bar{B}^{2}\right\rangle} \iint\left\|H\left(\mathbf{k}_{v}\right)\right\|^{2} \mathcal{R}_{\mathrm{t}}\left(\mathbf{k}_{v}\right) d^{2} k_{v},
\end{aligned}
$$

which is valid in general. For a given magnetized turbulence power spectrum $\mathcal{R}_{\mathrm{t}}\left(\mathbf{k}_{v}\right)$, the difference in $b^{2}(\ell=0)$ obtained with interferometry and single-dish observations resides in the nature of the filtering $H\left(\mathbf{k}_{v}\right)$ applied to the data. Although a determination of $b^{2}(\ell=0)$ with single dish will also be imprecise because of the spectral filtering at higher frequencies, the error is potentially more significant with interferometry since the spectral filtering is concentrated at low frequencies (see the "visibility" curves for Figures 2, 5, and 8) where the turbulent spectrum $\mathcal{R}_{\mathrm{t}}\left(\mathbf{k}_{v}\right)$ peaks. Evidently, the relative importance in these errors will depend on the precise shape of the corresponding single-dish and interferometer dirty beams, as well as that of the underlying spectrum. Moreover, the nature of the imprecision in the evaluation of $b^{2}(\ell=0)$ is made more complicated by the fact that $\left\langle\bar{B}^{2}\right\rangle \equiv\langle\overline{\mathbf{B}} \cdot \overline{\mathbf{B}}(0)\rangle$, present in the denominator of Equation (20), will also contain the same filtering integral as well as a similar one for the ordered component of the magnetic field. In some cases this may alleviate the aforementioned error, in others it may worsen it.

In the analysis of the SHARP OMC-1 data presented in Paper II, the effects of beam filtering and signal integration through the thickness of the cloud were corrected for by modeling the dispersion function while assuming circular Gaussian magnetized turbulent autocorrelation and beam functions. It is important to note, however, that even if it were possible to completely remove the filtering due to $\left\|H\left(\mathbf{k}_{v}\right)\right\|^{2}$ in Equation (20), the corresponding value obtained for $b^{2}(\ell=0)$ would still be underestimated because of the aforementioned signal integration along the line of sight. A correction for this effect would require a determination of the turbulent correlation length (see Paper II), which can be obtained by measuring the spectral width of $\mathcal{R}_{\mathrm{t}}\left(\mathbf{k}_{v}\right)$. More precisely, the turbulent correlation length is inversely proportional to the width of $\mathcal{R}_{\mathrm{t}}\left(\mathbf{k}_{v}\right)$. As this could only be achieved in general if $\mathcal{R}_{\mathrm{t}}\left(\mathbf{k}_{v}\right)$ is known at low frequencies (where it peaks), it is apparent that one would greatly benefit from combining single-dish and interferometry observations to maximize the spectral coverage at both ends of the spectrum.

\subsubsection{The Magnetized Turbulence Power Spectrum}

An inspection of the magnetized turbulence power spectrum $\mathcal{R}_{\mathrm{t}}(k) /\left\langle\bar{B}^{2}\right\rangle$ (or $\mathcal{R}_{1 \mathrm{D}}(k) /\left\langle\bar{B}^{2}\right\rangle$ ) shown in Figures 2 , 4 , and 8 makes it clear that we are able to determine its shape only at the high-frequency end, whereas it is expected that the inertial range of the power spectrum should extend over several decades in length scale (or spatial frequency). Taking the case of Orion KL as an example (see Figure 2), and acknowledging the fact that, as discussed in Section 4, our spectrum is unreliably estimated on the low-frequency end (i.e., for $k / 2 \pi \lesssim$ $0.05 \operatorname{arcsec}^{-1}$ ) we find that our analysis uncovers much less than a decade of the underlying spectrum (i.e., $0.07 \operatorname{arcsec}^{-1} \lesssim$ $k / 2 \pi \lesssim 0.2 \operatorname{arcsec}^{-1}$ ). One must therefore be cautious in putting too much weight in our determination of the scaling laws characterizing the small portion of the inertial range probed with our observations of Orion KL, IRAS 16293, and NGC 1333 IRAS 4A. Correspondingly, we once again emphasize the benefits that would thus be gained by combining single-dish and interferometry data. That is, a single-dish map of suitable spatial extent would ensure a good low-frequency coverage, while a high-resolution interferometry map of the same region would extend the measured turbulence power spectrum far enough to precisely characterize the inertial range as well as the turbulent ambipolar diffusion scale.

We should also keep in mind that the three-dimensional turbulence power spectrum underlying our data in Equation (10), i.e., $R_{3 \mathrm{D}, \mathrm{t}}(v, u)=\left\langle F(\mathbf{a}, z) F\left(\mathbf{a}^{\prime}, z^{\prime}\right)\right\rangle\left\langle\mathbf{B}_{\mathrm{t}}(\mathbf{a}, z) \mathbf{B}_{\mathrm{t}}\left(\mathbf{a}^{\prime}, z^{\prime}\right)\right\rangle$, contains the autocorrelation of the (ordered) polarized emission as well as that of the turbulent magnetic field. It therefore follows that the magnetized turbulence power spectrum we extract from our data does not exactly correspond to that of the turbulent magnetic field, but is somewhat broadened by the polarized emission spectrum. The importance of this effect may be advantageously investigated through numerical analyses and simulations.

\subsection{Further Improvements and Applications}

In all of our applications of the dispersion technique (i.e., in Papers I, II, and here), we always treated turbulence as being isotropic with a Kolmogorov-like power spectrum. This is, of course, a simplification that we do not expect to hold for magnetized turbulence in a weakly ionized plasma due to the anisotropy of motions in directions parallel and perpendicular to the magnetic field brought about by the Lorentz force. If, for example, one considers the theory of Goldreich \& Sridhar (1995) for incompressible magnetized turbulence, then different power-law scalings are expected for the power spectra measured along these two distinct orientations. Such anisotropy has, in fact, been measured in Taurus by Heyer et al. (2008) through ${ }^{12} \mathrm{CO}(J=1 \rightarrow 0)$ observations and optical polarization measurements using principal component analysis.

It would be straightforward in principle to extend our dispersion technique to allow for the detection of anisotropy. We would simply have to locally determine the mean orientation of the magnetic field about the position of a given datum on a polarization map and define, say, two sets of displacements $\ell_{\perp}$ and $\ell_{\|}$depending whether the distance vector $\ell$ linking that point to another one on the map is oriented more or less perpendicular or parallel to the mean magnetic field, respectively. Two dispersion analyses could then be performed, one for each of the $\ell_{\perp}$ and $\ell_{\|}$data sets. The main constraint in applying this technique for the polarization maps analyzed in this paper is the lack of data points. One needs a large number of points in order to accurately estimate the dispersion function. Moreover, it is also imperative that the change in orientation of the polarization vectors on a map varies smoothly enough that a mean direction for the magnetic field can be adequately calculated. Accordingly, 
we plan to attempt the implementation of this technique on the SHARP OMC-1 polarization map presented in Paper II in a future publication.

Another natural extension of this technique concerns the analysis of polarization maps of face-on spiral galaxies (see, e.g., the map of M51 presented in Fletcher et al. 2010). Although the polarization measured for external galaxies is not due to emission from anisotropic grains but from synchrotron radiation, we see no reason why the dispersion technique could not be applied to such cases. It would then also be natural to study the aforementioned anisotropy of magnetized turbulence since face-on spiral galaxies often show a mean magnetic field orientation that closely traces the spiral arms (Fletcher et al. 2010). Such analyses would also provide a detailed study of magnetized turbulence on a much larger scale than we have achieved so far (i.e., on galactic scales instead of that of molecular clouds).

\section{SUMMARY}

We presented an application of our magnetic field dispersion technique to high spatial resolution SMA polarization maps obtained for Orion KL in OMC-1, IRAS 16293, and NGC 1333 IRAS 4A. We showed how one can take advantage of such high-resolution data to characterize the magnetized turbulence power spectrum in the inertial and dissipation ranges. For Orion KL we determine that the inertial range of the spectrum approximately scales with $k^{-(2.9 \pm 0.9)}$ and we report a value of $9.9 \mathrm{mpc}$ for $\lambda_{\mathrm{AD}}$, the high spatial frequency cutoff presumably due to turbulent ambipolar diffusion. For the same parameters we have $\sim k^{-(1.4 \pm 0.4)}$ and a tentative value of $\lambda_{\mathrm{AD}} \simeq 2.2 \mathrm{mpc}$ for NGC 1333 IRAS 4A, and $\sim k^{-(1.8 \pm 0.3)}$ and an upper limit $\lambda_{\mathrm{AD}} \lesssim 1.8 \mathrm{mpc}$ for IRAS 16293 .

The authors thank the referee, P. M. Koch, for his valuable comments, which greatly improved this paper. M.H.'s research is funded through the NSERC Discovery Grant, Canada Research Chair, Canada Foundation for Innovation, Ontario Innovation Trust, and Western's Academic Development Fund programs.

\section{APPENDIX}

\section{WIENER FILTER AND DATA PROCESSING}

If we take into account the contribution of the spectral noise $n\left(\mathbf{k}_{v}\right)$ to $b^{2}\left(\mathbf{k}_{v}\right)$, we can write

$$
b^{2}\left(\mathbf{k}_{v}\right)=\hat{b}^{2}\left(\mathbf{k}_{v}\right)+n\left(\mathbf{k}_{v}\right)
$$

where the function

$$
\hat{b}^{2}\left(\mathbf{k}_{v}\right)=\left\|H\left(\mathbf{k}_{v}\right)\right\|^{2} \frac{\mathcal{R}_{\mathrm{t}}\left(\mathbf{k}_{v}\right)}{\left\langle\bar{B}^{2}\right\rangle}
$$

is assumed noiseless. The well-known solution for the Wiener filter to be applied to $b^{2}\left(\mathbf{k}_{v}\right)$ in order recover $\mathcal{R}_{\mathrm{t}}\left(\mathbf{k}_{v}\right) /\left\langle\bar{B}^{2}\right\rangle$ (in the least-squared sense) is given by Press et al. (1992)

$$
\phi\left(\mathbf{k}_{v}\right)=\frac{\left\|\hat{b}^{2}\left(\mathbf{k}_{v}\right)\right\|^{2} /\left\|H\left(\mathbf{k}_{v}\right)\right\|^{2}}{\left\|\hat{b}^{2}\left(\mathbf{k}_{v}\right)\right\|^{2}+\left\|n\left(\mathbf{k}_{v}\right)\right\|^{2}} .
$$

It is then necessary to somehow estimate the noise level $n\left(\mathbf{k}_{v}\right)$ and insert Equation (A2) into Equation (A3) to express the filter as a function of $\left\|H\left(\mathbf{k}_{v}\right)\right\|^{2}$ and the signal-to-noise ratio, as is often done. It is straightforward to show that the Wiener filter for our problem can be expressed as

$$
\phi\left(\mathbf{k}_{v}\right)=\frac{\left\|H\left(\mathbf{k}_{v}\right)\right\|^{2}}{\left\|H\left(\mathbf{k}_{v}\right)\right\|^{4}+\frac{\left\|n\left(\mathbf{k}_{v}\right)\right\|^{2}}{\left\|\mathcal{R}_{\mathrm{t}}\left(\mathbf{k}_{v}\right)\right\|^{2} /\left\langle\bar{B}^{2}\right\rangle^{2}}} .
$$

Equation (A4) is seen to tend to the obvious limit of $\phi\left(\mathbf{k}_{v}\right)=$ $1 /\left\|H\left(\mathbf{k}_{v}\right)\right\|^{2}$ when $n\left(\mathbf{k}_{v}\right)$ vanishes. We compute our Wiener filter by (1) determining the mean level $\bar{n}$ for $n\left(\mathbf{k}_{v}\right)$ in the high-frequency end of the spectrum for $b^{2}\left(\mathbf{k}_{v}\right)$ where $\hat{b}^{2}\left(\mathbf{k}_{v}\right)$ is negligible, (2) subtracting $\bar{n}$ from $b^{2}\left(\mathbf{k}_{v}\right)$ to approximately obtain $\mathcal{R}_{\mathrm{t}}\left(\mathbf{k}_{v}\right) /\left\langle\bar{B}^{2}\right\rangle$, and (3) inserting $\|\bar{n}\|^{2}$ (in lieu of $\left\|n\left(\mathbf{k}_{v}\right)\right\|^{2}$ ) and $\left\|\mathcal{R}_{\mathrm{t}}\left(\mathbf{k}_{v}\right)\right\|^{2} /\left\langle\bar{B}^{2}\right\rangle^{2}$ in Equation (A4).

Finally, we note that we processed the data in the spectral domain using discrete Fourier transforms (DFTs). Although the shapes of the $b^{2}(\ell)$ functions shown in Figures 1, 4, and 7 are such that they tend to smoothly approach zero at the larger values of $\ell$, the presence of noise and of residual levels can potentially be the cause of "edge effects" and ensuing contamination of the spectra $b^{2}\left(\mathbf{k}_{v}\right)$. We have therefore windowed the data and the synthesized beam in the $\ell$ domain with a Hanning window (Hamming 1997) before applying DFTs in order to minimize these effects.

\section{REFERENCES}

Brogan, C. L., \& Troland, T. H. 2001, ApJ, 560, 821

Chandrasekhar, S., \& Fermi, E. 1953, ApJ, 118, 113

Crutcher, R. M., Hakobian, N., \& Troland, T. H. 2010, MNRAS, 402, L64

Crutcher, R. M., Troland, T. H., Lazareff, B., Paubert, G., \& Kazès, I. 1999, ApJ, 514, L121

Falceta-Gonçalves, D., Lazarian, A., \& Houde, M. 2010, ApJ, 713, 1376

Falgarone, E., Troland, T. H., Crutcher, R. M., \& Paubert, G. 2008, A\&A, 487, 247

Fletcher, A., Beck, R., Shukurov, A., Berkhuijsen, E. M., \& Horellou, C. 2010, MNRAS, 412, 2396

Frisch, U. 1995, Turbulence: The Legacy of A. N. Kolmogorov (Cambridge: Cambridge Univ. Press)

Girart, J. M., Rao, R., \& Marrone, D. P. 2006, Science, 313, 812

Goldreich, P., \& Sridhar, S. 1995, ApJ, 438, 763

Hamming, R. W. 1997, Digital Filters (3rd ed; Mineola, NY: Dover)

Heiles, C. 1997, ApJS, 111, 245

Heyer, M., Gong, H., Ostriker, E., \& Brunt, C. 2008, ApJ, 680, 420

Hezareh, T., Houde, M., McCoey, C., \& Li, H. 2010, ApJ, 720, 603

Hildebrand, R. H., Kirby, L., Dotson, J. L., Houde, M., \& Vaillancourt, J. E. 2009, ApJ, 696, 567 (Paper I)

Ho, P. T. P., Moran, J. M., \& Lo, K. Y. 2004, ApJ, 616, L1

Houde, M., Bastien, P., Peng, R., Phillips, T. G., \& Yoshida, H. 2000, ApJ, 536, 847

Houde, M., Vaillancourt, J. E., Hildebrand, R. H., Chitsazzadeh, S., \& Kirby, L. 2009, ApJ, 706, 1504 (Paper II)

Houde, M., Hezareh, T., Li, H., \& Phillips, T. G. 2011, Mod. Phys. Lett. A, 26, 235

Koch, P. M., Tang, Y.-W., \& Ho, P. T. P. 2010, ApJ, 721, 815

Lazarian, A., Vishniac, E. T., \& Cho, J. 2004, ApJ, 603, 180

Li, H., \& Houde, M. 2008, ApJ, 677, 1151

Marrone, D. P., Moran, J. M., Zhao, J.-H., \& Rao, R. 2006, ApJ, 640, 308

Marrone, D. P., \& Rao, R. 2008, Proc. SPIE, 7020, 70202B

Mouschovias, T. Ch., \& Tassis, K. 2009, MNRAS, 400, L15

Press, W. H., Flannery, B. P., Teukolsky, S. A., \& Vetterling, W. T. 1992, Numerical Recipes in C: The Art of Scientific Computing (2nd ed.; Cambridge: Cambridge Univ. Press)

Rao, R., Girart, J. M., Marrone, D. P., Lai, S.-P., \& Schnee, S. 2009, ApJ, 707, 921

Tang, Y.-W., Ho, P. T. P., Koch, P. M., \& Rao, R. 2010, ApJ, 717, 1262

Tilley, D. A., \& Balsara, D. S. 2010, MNRAS, 406, 1201

Wright, M. C. H., \& Sault, R. J. 1993, ApJ, 402, 546 